\title{
IRAK4 gene polymorphism and odontogenic maxillary sinusitis
}

\author{
Inês Guerra Pereira • Paula Vaz • Ricardo Faria Almeida • \\ Ana Cristina Braga • António Felino
}

Received: 10 October 2014 / Accepted: 2 February 2015 / Published online: 25 February 2015

(C) Springer-Verlag Berlin Heidelberg 2015

\begin{abstract}
Objectives This study aimed to evaluate whether a specific interleukin-1 receptor-associated kinase-4 (IRAK4) gene polymorphism had any influence on the development of changes in maxillary sinus, particularly in the presence of etiological factors of dental origin.

Materials and methods The study population included 153 Portuguese Caucasians that were selected from a database of 504 retrospectively analysed computed tomography (CT) scans. A genetic test was performed, and a model was created through logistic analysis and regression coefficients. The statistical methodologies included were the independent Chi test, Fisher's exact test, binary logistic regression and the receiver operating characteristic (ROC) curve.

Results The estimated prevalence of IRAK4 gene polymorphism found in a Portuguese Caucasian population was 26.8 $\%$ (CI $95 \%$ ) [20.1, $34.7 \%$. A model to predict the inflam-
\end{abstract}

\footnotetext{
I. G. Pereira $(\bowtie) \cdot$ R. F. Almeida $\cdot$ A. Felino

Department of Oral Surgery, Faculty of Dentistry, Oporto University,

Rua Dr. Manuel Pereira da Silva, 4200393 Porto, Portugal

e-mail: inesguerrapereira@gmail.com

R. F. Almeida

e-mail: ralmeida@fmd.up.pt

A. Felino

e-mail: afelino@fmd.up.pt

P. Vaz

Department of Orofacial Genetics, Faculty of Dentistry, Oporto University, Porto, Portugal

e-mail: pvaz@fmd.up.pt

A. C. Braga

Department of Production and Systems Engineering, University of

Minho, Braga, Portugal

e-mail: acb@dps.uminho.pt
}

matory response in the maxillary sinus in the presence etiological factors of dental origin was constructed. This model had the following as variables: previously diagnosed sinusitis, sinus pressure symptoms, cortical bone loss observed on CT, positive genetic test result and radiographic examination that revealed the roots of the teeth communication with the maxillary sinus, which are interpreted as risk factors.

Conclusions The constructed model should be considered an initial clinical tool. The area under the ROC curve found, $\mathrm{AUC}=0.91$, revealed that the model correctly predicts the outcome in $91.1 \%$ of cases.

Clinical relevance The clinical relevance of this study lies in trying to achieve a potential tool (a model) that may assist the clinician in the implementation of suitable dental treatment plans in complex cases, with probable involvement of the maxillary sinus.

Keywords Genetic polymorphism · Maxillary sinusitis · IRAK-4 human protein · Genetic predisposition

\section{Introduction}

Inflammatory sinus disease is the most common pathological condition involving the paranasal sinuses, and the chronic inflammatory disease of undefined etiopathogenesis of the upper airways is considered to affect 6 to $13 \%$ of the population [1,2].

All forms of maxillary sinusitis (more specific chronic sinusitis) were related with loss of an anatomic barrier and with innate immune response. The latter normally prevents infection of healthy sinuses, however, in some patients, this prevention is ineffective and they may be considered highly predisposed to trigger repeated clinical conditions of acute 
sinusitis. Added to this, maxillary sinusitis was associated with the loss of sterility (observed in healthy sinuses) and was routinely linked with the presence of gram-negative aerobic bacteria - Staphylococcus aureus - and other anaerobic bacteria $[3,4]$. In fact, the classic odontogenic infection of the maxillary sinus is composed of a mixed aerobic-anaerobic infection, with anaerobes predominating [2]. Therefore, several authors support the theory that oral bacteria may ascend from the mouth to the maxillary sinus and consequently be the cause of sinus infections [1, 2, 4-8]. This type of maxillary sinusitis - odontogenic - occurs when the Schneider membrane is violated. Also, since the very beginning of investigation about the etiology of the maxillary sinusitis, dental factors have been associated to $10-12 \%$ cases of chronic maxillary sinusitis [7].

The oral conditions that were related with the odontogenic infection of the sinus include infections of the maxillary posterior teeth (periapical granulomas caused by caries, periodontal disease, cysts roots or incorrect endodontics procedures), oroantral fistula, pathologic lesions of the jaws and teeth, maxillary dental trauma or iatrogenic causes, such as sinus foreign bodies (dental fillings, teeth roots, broken instruments or even dental implants) $[1,6,9]$.

The role played by host inflammatory response in the maxillary sinus disease has been called into question for investigation for more than a decade. Although, it is believed that this response may be controlled by specific genes, which determine a more or less pronounced phenotypic scene disease [2, 3, 10-12].

In fact, specific gene polymorphisms [base-pair substitutions in the DNA sequence, frequently called single nucleotide polymorphisms (SNPs)] have been proved to interfere with the innate pathway of immune recognition and with the regulation of the host response. The latter relation has been also suggested for the maxillary sinusitis and rhinitis pathway [2, 3, 12-14].

Some studies have reported a relation between specific genetic variations in the interleukin-1 receptor-associated kinase-4 (IRAK4) gene, the host susceptibility to develop rhinitis and the prevalence of Gram-positive bacterial infections in critically ill adults [13, 14]. Other investigations have also added the existence of a relationship between Gram-positive recurrent infections and deficient defence reactions in paediatric patients with genetic IRAK4 mutations [15-17].

IRAK4 is a serine-threonine kinase that is considered a key player in the signalling of the MyD88-depending pathway of the Toll-like/IL-1-receptor (TIR) and also in other Toll-like receptor (TLR) signalling, except TLR3 [18]. When the IRAK4 gene interacts with MyD88, it leads to the activation of the nuclear factor- $k b$ (NF-KB) and, consequently, to the transcription of inflammatory mediators (such as IL-6) [18].
Zhang et al. (2011) reported a gender- and allergendependent association pattern between some IRAK4 gene polymorphisms and allergic rhinitis in a Chinese population.

Moreover, the Sutherland and co-workers study (2011) suggested that the IRAK4 gene haplotype, clade marked by 29429A (428Thr), modify the susceptibility to Gram-positive bacteria infection, by decreasing cellular response to TLR ligands.

It is probable that genetic variation alone does not create disease as such, but, instead confers susceptibility to its development, just as has been suggested for the host response in other fields of oral intervention [19]. Thus, this predisposition to the disease development may require an interaction with an environmental factor to yield disease [2].

According to Toby Hughes, genetic research means we can use it to determine the health status of individuals or even to influence how genes behave. So, there is the possibility to early monitor potential oral diseases in order to prevent or reduce their impact $[19,20]$.

This study analysed IRAK4 gene polymorphism [marked by 29429A (428Thr)] in a Portuguese Caucasian population. The clinical relevance of this study lies in trying to achieve a potential tool (a model) that may assist the clinician in the implementation of suitable dental treatment plans in complex cases, with probable involvement of the maxillary sinus.

The aim was to determine whether the haplotype clade marked by 29429A (428Thr) polymorphism had any influence on the development of changes in the maxillary sinus, particularly in the presence of etiological factors of dental origin.

\section{Material and methods}

IRAK4 gene was selected as a candidate gene for this investigation based on its central function in innate and acquired immunity. The election of a specific IRAK4 polymorphism was performed according to the host response against the oral flora.

\section{Study subjects}

A retrospective study review was initially conducted to identify and characterize computed tomography (CT) imaging findings of a Portuguese Caucasian population. Institutional Review Board approval was obtained, and appropriate measures were taken to safeguard patient privacy.

The CT scans were performed according to the following indications: implant planning, study of impacted maxillary teeth, suspected lesion/cyst on the maxillary sinus, ankylosis of the anterior teeth, suspected foreign body in the maxillary sinus, endodontic lesions, oroantral fistula, orthodontic planning, local trauma and dental or bone fractures. 
The criteria for patient selection were based on etiological factors of the maxillary sinusitis of odontogenic origin and the pathologic changes present in the maxillary sinus [21-23]. The CT's observed were from the period between 1993 and 2013. Two observers with over 20 years experience in the field of oral surgery identified the eventual changes present on CT.

In the analysed maxillary sinus, only the adjacent teeth to the maxillary sinus were evaluated [22-28] and the following signs were identified - opacity, presence of fluid, mucosal thickening, cortical bone loss [21,29] and potential etiologic factors to develop sinusitis of odontogenic origin - teeth protruding into the maxillary sinus (maxillary sinus communication), dental caries, periodontal disease, apical periodontitis, endodontic treatment, iatrogenic signs, dental implants, cysts, foreign bodies, ectopic teeth, oroantral fistula and inclusion of teeth associated with maxillary sinus.

These completed a total of 504 patients, corresponding to 1008 analysed maxillary sinus.

From this base, 153 patients were randomly selected, according to the value of $13 \%$ for the maxillary sinusitis prevalence, with $95 \% \mathrm{CI}$ and estimated error of $5.3 \%$ to perform a genetic test.

According to the classification of Maillet et al. (2011), the patients were divided and included into four groups:

Group IA: Presence of imagiological findings suggesting sinusitis of odontogenic origin - density of soft tissue mass or air-fluid within the sinus and presence of one of the following criteria: decayed tooth, tooth restoration faulty and extraction site with mucosal thickening. Forty-six (46) patients were identified in this group.

Group IB: The sinus was considered healthy in the absence of mucosal thickening or uniform mucosal thickening less than $2 \mathrm{~mm}$. Also, adjacent teeth should evidence signs of carious lesions, decayed tooth, exposed pulp, restoration, extraction and have imaging of apical periodontitis (presence of potential etiologic odontogenic factors). Thirty-four (34) patients were identified as having at least one of this potential etiologic odontogenic factors.

Group IIA: Presence of soft tissue mass within the sinussinusitis of non-odontogenic origin, having fulfilled the following criteria: healthy teeth, coronal restoration and/or endodontic good quality, absence of periapical lesion, tooth extracted intact or healed alveoli and thickening of the mucosa not limited to any tooth. Thirty-five (35) patients were identified with this condition.

Group IIB: Healthy patients - absence of mucosal thickening or uniform mucosal thickening less than $2 \mathrm{~mm}$ defective, having fulfilled the following criteria: healthy teeth, coronal restoration and/or endodontic good quality, absence of periapical lesion, tooth extracted intact or healed alveoli and thickening of the mucosa not limited to any tooth (absence of potential etiologic odontogenic factors). Thirty-eight (38) patients were considered in this group.

The genetic test was performed in the same appointment where the baseline demographics and medical data were recorded: familiar history of sinusitis, past maxillary sinus surgery, sinusitis or rhinitis diagnosed by an otolaryngologist, actual drug prescription, asthma or diabetes, smoking habit and presence of symptoms according to the Rosenfeld (2007) criteria-headache, nasal obstruction (congestion), ear pain, mucopurulent drainage, decreased sense of smell and dental pain. Intra-oral examination was carried out concerning facial pain on percussion or palpation, facial edema and erythema.

\section{Inclusion criteria}

All adult Caucasian patients ( $>18$ years) who had at least one CT scan performed were included in this study. The patients with immune-compromised conditions, patients with CT without sufficient slices or poor quality image to analyze the maxillary sinus and patients with $\mathrm{CT}$ that did not allow analyzing the maxillary teeth were excluded.

\section{Selection of polymorphism in the human IRAK4 gene}

The IRAK4 gene analysis was initially performed by sequencing all gene (Fig. 1). The latter was done by polymerase chain reaction (PCR) amplification and sequencing of the entire coding region, including the adjacent intronic regions of the IRAK4 gene. In this first analysis, pathogenic mutations were not detected in the IRAK4 gene. However, the variant c. $1882 \mathrm{G}>\mathrm{A}$ (p.Ala428Thr) was found in heterozygosity (a single nucleotide polymorphism-SNP). This SNP was the same reported to be related with the host susceptibility for development of Gram-positive bacteria infection [13].

After this SNP identification, the following samples were studied directly target to the c.1282G $>$ A (p.Ala428Thr) variant region (reference sequence: NM_001114182.2, being the A of the ATG start position 1). The primers selected to detect the SNP on the IRAK4 gene are present in Table 1. Furthermore, the D (down) was considered the forward, the U (up) the reverse and the 12/13 were together in just a fragment (because it was smaller and likely to be amplified with a single primer pair region). 
Fig. 1 Sequencing image revealing a IRAK4 gene polymorphism, translated by two peaks, indicated by the arrow and identified in a recruited patient study

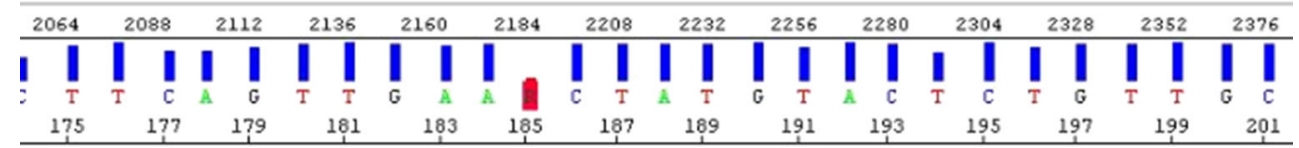

The genotypic analysis was performed in a blinded fashion, without clinical information. Patients' genotypes were determined by real-time PCR and the DNA sequencing resorted to the Sanger method.
Table 1 Primers used to detect the SNP on the IRAK4 human gene [Ref Seq: NM_016123]

\begin{tabular}{ll}
\hline Primer & Sequence \\
\hline IRAK4-3D & TGTAAAACGACGGCCAGTAAAGGAAGCAAACCCAGAGG \\
IRAK4-3U & CAGGAAACAGCTATGACCGGGAACCACAGCAAAGGTG \\
IRAK4-4D & TGTAAAACGACGGCCAGTGCCTCTCGATCTTGGACTTCC \\
IRAK4-4U & CAGGAAACAGCTATGACCCACAGTGTCCTTTGTCTGTGC \\
IRAK4-5D & TGTAAAACGACGGCCAGTCTCTGATATAGGTGGCTCATGG \\
IRAK4-5U & CAGGAAACAGCTATGACCTGCCTGTGATTGCTGCAC \\
IRAK4-6D & TGTAAAACGACGGCCAGTGTTCAGAGGGTAGGATCACC \\
IRAK4-6U & CAGGAAACAGCTATGACCGGTAGGGTCCATAGTGTTCAAC \\
IRAK4-7D & TGTAAAACGACGGCCAGTATGGGCCTTGAGCAAATCAC \\
IRAK4-7U & CAGGAAACAGCTATGACCCCTGTAGGTCCAGCTACTCG \\
IRAK4-8D & TGTAAAACGACGGCCAGTGGCTGAAAAGAGAAGTATTTGC \\
IRAK4-8U & CAGGAAACAGCTATGACCAATGGCAACCCAGTTGTTG \\
IRAK4-9D & TGTAAAACGACGGCCAGTATCATCTTCAGTTGTTGCCTAG \\
IRAK4-9U & CAGGAAACAGCTATGACCGAGCTTTCATGGAGTGATGG \\
IRAK4-10D & TGTAAAACGACGGCCAGTAAAGAGGACAGTTGCTTCTC \\
IRAK4-10U & CAGGAAACAGCTATGACCCCCACACCTATCTCTTGCTC \\
IRAK4-11D & TGTAAAACGACGGCCAGTATCTGTCTCCCAAAGTGCTG \\
IRAK4-11U & CAGGAAACAGCTATGACCCTGTCGTGTAATAGCCAATGG \\
IRAK4-12D & TGTAAAACGACGGCCAGTGAGCACATGTTATTACAAAGTAG \\
IRAK4-13U & CAGGAAACAGCTATGACCGTGTTTCCACCCTACATACAG \\
\hline
\end{tabular}


Statistical analysis

Using the software IBM ${ }^{\circledR}$ SPSS ${ }^{\circledR}$ Statistics 22.0, the sample was characterized, an analytical study of association was conducted and data modelling was performed using the binary logistic regression, for dependent variable "diagnosis" corresponding to two categories, one for healthy individuals and the other for individuals with sinusitis of odontogenic origin. With this, the odds ratio due to the presence or absence of certain risk factor in this sample was evaluated.

After a univariate selection of variables, the construction of a model proceeded. In the construction of the logistic regression model, the forward stepwise technique was used to optimize variable selection, in order to select a set of variables that could contribute to the outcome and that this contribution revealed statistically significant. Therefore, in the previous univariate analysis, we took into account the $p$ values obtained in the Wald statistic, and these variables enter as candidates to integrate the model.

The objective was to achieve a model that contained all variables that were important with regard to pre-established criteria of $\mathrm{pE}$ and $\mathrm{pR}$ values ( $p$ values of entry and removal of the variable in the model) chosen so that they become statistically and clinically significant. In other words, a variable could not present a $p$ value $<0.05$ but may be selected to include the model. Sometimes, a variable cannot have a significant effect individually, but in a particular set potentiate its effect on the outcome variable. That was performed taking into account the importance of the selected variable in the recent literature outlines and in the clinical setting.

Categorical variables were coded according to the technique referred by Hosmer and Lemeshow $[30,31]$.

Receiver operating characteristic (ROC) analysis is a useful tool for evaluating the performance of diagnostic tests and more generally for evaluating the accuracy of a statistical model (e.g. logistic regression, linear discriminant analysis) that classifies subjects into one of two categories, diseased or non-diseased [19, 31]. Using a graphical tool, ROC curve, and an accuracy measure such as the area under ROC curve, is in our days used for displaying the accuracy of a predictive model to estimate expected outcomes. So, to evaluate the accuracy of our model, we use ROC curve and its measure area under curve (AUC).

\section{Results}

The sample consisted of 153 individuals, of which 62 $(40.5 \%)$ were female and the remaining 91 (59.5\%) were male.

The prevalence of the polymorphism c. $1282 \mathrm{G}>\mathrm{A}$ (p.Ala428Thr) in the variant region of the IRAK4 gene in this

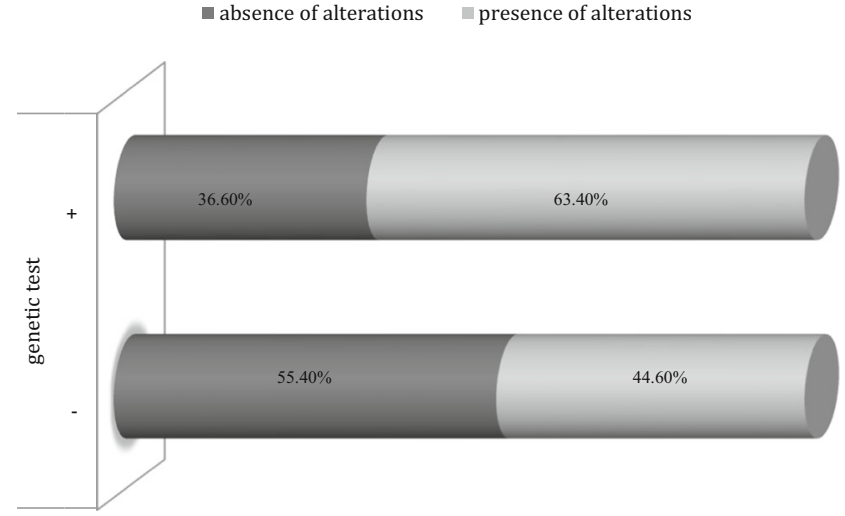

Fig. 2 Illustration of the statistical association between the presence of IRAK4 gene polymorphism (positive genetic test) and the presence of the mucosal thickening of maxillary sinus on computed tomography image

sample was $26.8 \%$, with a confidence interval of $95 \%$ [20.1, $34.7 \%$ ].

The Fisher exact test $(p=0.030<0.05)$ showed statistically significant association between the presence of the IRAK4 gene polymorphism and the mucosal thickening on CT imaging (Fig. 2).

The Fisher exact test $(p=0.040<0.05)$ revealed statistically significant association between the presence of the IRAK4 gene polymorphism and the diagnosis of sinusitis of odontogenic origin (Fig. 3).

Fisher's exact test was performed in order to determine a possible statistical relationship between the presence of the IRAK4 gene polymorphism and the presence of diabetes or asthma. No significant associations were found.

Regarding the symptoms related to the diagnosis of maxillary sinusitis, there was no statistically significant association ( $p>0.05)$ between the IRAK4 polymorphism and the symptoms individually (Table 2).

Also, a statistically significant association $(p>0.05)$ between the IRAK4 polymorphism and the different characteristics evaluated in the physical examination was not found (Table 3).

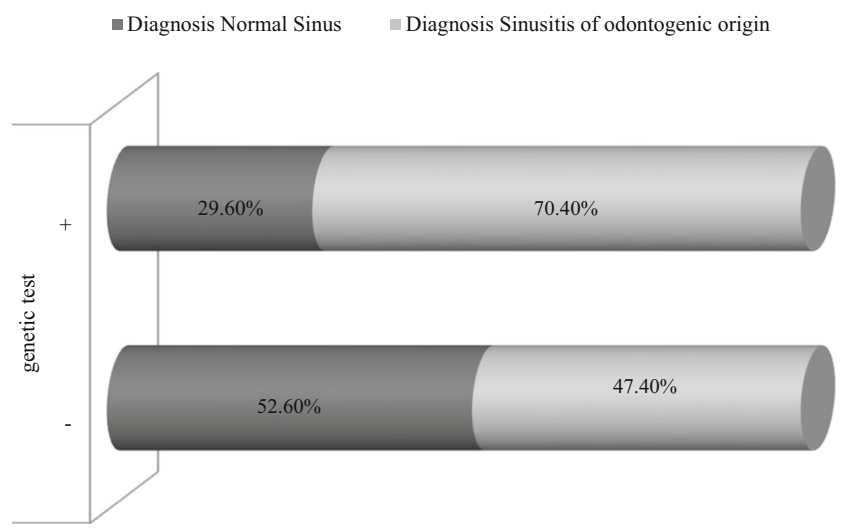

Fig. 3 Relation between the IRAK4 gene polymorphism (positive genetic test) and maxillary sinusitis of odontogenic origin 
Table 2 Results of the Fisher exact test for association between the IRAK4 gene polymorphism and patient symptoms

\begin{tabular}{lc}
\hline Patient symptoms & $p$ value \\
\hline Headache & 0.426 \\
Mucopurulent drainage & 0.342 \\
Nasal obstruction & 0.393 \\
Decreased sense of smell & 0.525 \\
Ear pain & 0.478 \\
Dental pain & 0.331 \\
\hline
\end{tabular}

As for the relation between the patient medication and the presence of evaluated genetic polymorphism (Table 4), there was no statistically significant association $(p>0.05)$.

The final sample to construct the prevision model was accomplished through the selection of two groups of the main sample (Groups IIB and IA). So, the latter consisted of 84 individuals, including 38 (45.2\%) (Group IIB) healthy and the remaining 46 were diagnosed with sinusitis of odontogenic origin (54.8\%) (Group IA).

The prevalence of the polymorphism c. $1282 \mathrm{G}>\mathrm{A}$ (p.Ala428Thr) in the variant region of the IRAK4 gene in this final sample was $32.1 \%$, with a confidence interval of $95 \%$ [22.6, $43.3 \%]$.

Thirty-four (40.5\%) of the individuals in this final sample were female, and the remaining $50(59.5 \%)$ were male. The Fisher exact test revealed that the distribution by sex according to the diagnosis was the same $(p=0.826)$ in the final sample. The age distribution in the final sample is shown in Table 5.

The Student $t$ test showed no statistically significant difference in the mean age of subjects in the two groups $(t(82)=$ $-0.238, p=0.812$ ) of the final sample.

In a first step of selecting variables to include in the model, the dependent variable was elected, which is the diagnosis (codes: $0=$ healthy, $1=$ sinusitis of odontogenic origin). Then, we proceeded to the univariate analysis by binary logistic regression. Thus, taking into account the $P$ values obtained in the test of Wald statistics, the variables were considered as candidates to integrate the model indicated in Table 6.

The variables marked with a number sign in Table 6 indicate instability due to the high value of the standard error, and were not considered for analysis. The values marked with an asterisk indicate that there is a statistically significant

Table 3 Results of the Fisher exact test for association between the $I R A K 4$ gene polymorphism and the evaluated parameters in the physical examination

\begin{tabular}{lc}
\hline Physical examination & $p$ value \\
\hline Palpation pain & 0.220 \\
Edema & 0.389 \\
Erythema & 0.283 \\
\hline
\end{tabular}

Table 4 Results of

Fisher exact test for association between the medication and the presence of IRAK4 gene polymorphism

\begin{tabular}{ll}
\hline Medication & $p$ value \\
\hline Anticoagulants & 0.457 \\
Corticosteroids & 0.489 \\
Antihypertensives & 0.18 \\
Anti-depressants & 0.606 \\
Antibiotics & 0.604 \\
Anti-inflammatory & 0.17 \\
\hline
\end{tabular}

association of this variable with the diagnosis groups. So, every variable, with this result, was a good candidate to integrate the multivariate model. Therefore, from the univariate logistic regression results, the following candidate variables to enter the model - diagnosed sinusitis, nasal congestion, cortical bone loss, genetic test and maxillary sinus communication-were selected.

Using the forward conditional stepwise (with $\mathrm{pE}=0.2$ and $\mathrm{pR}=0.25$ ), we obtained a decision model in order to predict the result according to presence of a set of considered risk factors [30]. The choice of $\mathrm{pE}$ and $\mathrm{pR}$ was made in accordance with Hosmer and Lemeshow that recommend, in models containing more variables, the use of a large $p$ value to provide a more complete picture of possible models. The results obtained were summarized in Table 7.

To evaluate the discriminatory power of the model to predict the result, we used the measured area under the ROC curve (AUC) [32]. Figure 4 represents the empirical ROC curve to the estimated probabilities to the decision model.

The area under the curve AUC $=0.911$ means that the model could predict the outcome in $91.1 \%$ of cases.

In multivariate logistic regression model, the probability estimated by the model, incorporating the $p$ variables, can be written by the expression:

$\widehat{\pi}\left(x_{i}\right)=\frac{\exp \left(B_{0}+B_{1} x_{1}+\ldots+B_{p} x_{p}\right)}{1+\exp \left(B_{0}+B_{1} x_{1}+\ldots+B_{p} x_{p}\right)}$

Thus, for the proposed model, an individual without sinusitis diagnosed, without complaints of feeling nasal congestion in the region of the maxillary sinuses, with cortical bone loss

Table 5 Age distribution according to diagnosis in the final sample

\begin{tabular}{lllll}
\hline & \multicolumn{3}{l}{ Diagnostic } & \\
\cline { 3 - 5 } & & Healthy & Odontogenic sinusitis & Total \\
\hline \multirow{2}{*}{ Age (years) } & Mean & 42.0 & 42.8 & 42.5 \\
& Median & 41.5 & 43.5 & 42.0 \\
& Standard deviation & 15.9 & 14.0 & 14.8 \\
& Minimum & 18.0 & 18.0 & 18.0 \\
\hline
\end{tabular}


Table 6 Univariate logistic regression

\begin{tabular}{|c|c|c|c|c|c|c|}
\hline Variables & $B$ & S.E. & Wald & df & Sig. & $\operatorname{Exp}(\mathrm{B})$ \\
\hline Gender & 0.123 & 0.446 & 0.076 & 1 & 0.782 & 1.131 \\
\hline Family history of maxillary sinusitis & 1.959 & 0.797 & 6.042 & 1 & $0.014^{*}$ & 7.091 \\
\hline Motive of dental appointment & 0.916 & 0.453 & 4.088 & 1 & $0.043^{*}$ & 2.500 \\
\hline Maxillary sinusitis & 2.982 & 1.060 & 7.921 & 1 & $0.005^{*}$ & 19.733 \\
\hline Allergic rhinosinusitis & 2.330 & 1.075 & 4.701 & 1 & $0.030^{*}$ & 10.278 \\
\hline Diabetes & -0.951 & 0.896 & 1.127 & 1 & 0.288 & 0.386 \\
\hline Tobacco & 0.730 & 0.591 & 1.522 & 1 & 0.217 & 2.074 \\
\hline Headache & 2.356 & 0.788 & $8.939^{*}$ & 1 & $0.003^{*}$ & 10.552 \\
\hline Pressure & 3.169 & 1.057 & $8.981^{*}$ & 1 & $0.003^{*}$ & 23.786 \\
\hline Ear pain & 21.285 & $12,118.636$ & 0.000 & 1 & $0.999^{\#}$ & $1,753,944,115.096$ \\
\hline Mucopurulent drainage & 1.878 & 0.607 & 9.584 & 1 & $0.002^{*}$ & 6.538 \\
\hline Decreased sense of smell & 21.375 & $10,742.023$ & 0.000 & 1 & $0.998^{\#}$ & $1,918,376,375.886$ \\
\hline Dental pain & 21.314 & $11,602.711$ & 0.000 & 1 & $0.999^{\#}$ & $1,805,530,706.716$ \\
\hline Pain on percussion & 21.375 & $10,742.023$ & 0.000 & 1 & $0.998^{\#}$ & $1,918,376,375.886$ \\
\hline Pain on palpation & 2.330 & 1.075 & $4.701^{*}$ & 1 & $0.030^{*}$ & 10.278 \\
\hline Swelling/tumefaction & 21.285 & $12,118.636$ & 0.000 & 1 & $0.999^{\#}$ & $1,753,944,115.096$ \\
\hline Erythema & 21.079 & $23,205.422$ & 0.000 & 1 & $0.999^{\#}$ & $1,427,628,930.892$ \\
\hline Dentate behaviour on CT (computed tomography) & -21.476 & $23,205.422$ & 0.000 & 1 & $0.999^{\#}$ & 0.000 \\
\hline Maxillary sinus communication & 2.606 & $0.552^{*}$ & 22.248 & 1 & $0.000^{*}$ & 13.538 \\
\hline Dental implants & 21.152 & $16,408.711$ & 0.000 & 1 & $0.999^{\#}$ & $1,534,701,100.709$ \\
\hline Dental decay & 21.056 & $28,420.721$ & 0.000 & 1 & $0.999^{\#}$ & $1,395,182,818.826$ \\
\hline Periodontal disease & 21.103 & $20,096.485$ & 0.000 & 1 & $0.999^{\#}$ & $1,461,620,095.913$ \\
\hline Periapical lesion & 21.545 & 9220.900 & 0.000 & 1 & $0.998^{\#}$ & $2,273,631,260.309$ \\
\hline Endodontic treatment & 21.545 & 9220.900 & 0.000 & 1 & $0.998^{\#}$ & $2,273,631,260.309$ \\
\hline Cysts & 21.177 & $15,191.515$ & 0.000 & 1 & $0.999^{\#}$ & $1,574,052,410.983$ \\
\hline Oroantral fistula & 21.034 & $40,192.969$ & 0.000 & 1 & $1.000^{\#}$ & $1,364,178,756.185$ \\
\hline Impacted teeth & 21.406 & $10,377.780$ & $0.000^{\#}$ & 1 & $0.998^{\#}$ & $1,980,259,484.785$ \\
\hline Foreign bodies & 21.034 & $40,192.969$ & $0.000^{\#}$ & 1 & $1.000^{\#}$ & $1,364,178,756.185$ \\
\hline Radiopacity & 22.068 & 7338.199 & $0.000^{\#}$ & 1 & $0.998^{\#}$ & $3,836,752,751.771$ \\
\hline Presence of air fluid & 21.152 & $16,408.711$ & $0.000^{\#}$ & 1 & $0.999^{\#}$ & $1,534,701,100.709$ \\
\hline Mucosal thickening & 24.147 & 6059.318 & $0.000^{\#}$ & 1 & $0.997^{\#}$ & $30,694,022,014.172$ \\
\hline Cortical bone loss & 4.240 & 1.060 & 16.007 & 1 & $0.000^{*}$ & 69.375 \\
\hline Implant lesion & 21.079 & $23,205.422$ & 0.000 & 1 & $0.999^{\#}$ & $1,427,628,930.892$ \\
\hline Genetic test & 0.970 & 0.498 & 3.797 & 1 & $0.051^{*}$ & 2.639 \\
\hline
\end{tabular}

* Indicates that there is a statistically significant association of this variable with the diagnosis groups

${ }^{\#}$ Indicates instability due to the high value of the standard error, and was not considered for analysis

observed on CT, with a positive genetic test result and radiographic examination that reveals the roots of the teeth in communication with the maxillary sinus (maxillary sinus communication), it is estimated, by the model, a probability value of 0.977 , to triggering an inflammatory response in the maxillary sinus.

\footnotetext{
$\pi\left(x_{i}\right)=\frac{\exp (-2.121+2.241 * 0+2.367 * 0+3.635 * 1+1.239 * 1+1.01 * 1)}{1+\exp (-2.121+2.241 * 0+2.367 * 0+3.635 * 1+1.239 * 1+1.01 * 1)}$ $=0.977$
}

\section{Discussion}

Currently, chronic diseases are a major cause of morbidity and mortality worldwide, and a large proportion of chronic diseases are preventable through risk factor management. Chronic disease prediction models have been developed to assist physicians/clinicians and individuals in clinical decision-making. A chronic disease prediction model assesses multiple risk factors together and estimates an absolute disease risk for the individual. In the selection of the variables to include a model, as suggested by Hosmer and Lemeshow, 
Table 7 Variables in the equation resulted from forward conditional stepwise

\begin{tabular}{lcccccc}
\hline Variables & $B$ & S.E. & Wald & Df & $p$ value & Exp (B) \\
\hline Diagnosed sinusitis & 2.341 & 1.257 & 3.471 & 1 & 0.062 & 10.396 \\
Nasal congestion & 2.367 & 1.248 & 3.596 & 1 & 0.058 & 10.666 \\
Cortical bone loss & 3.635 & 1.202 & 9.146 & 1 & 0.002 & 37.898 \\
$\begin{array}{l}\text { IRAK4 gene } \\
\text { polymorphism }\end{array}$ & 1.239 & 0.745 & 2.765 & 1 & 0.096 & 3.453 \\
$\begin{array}{c}\text { Maxillary sinus } \\
\quad \text { communication }\end{array}$ & 1.010 & 0.833 & 1.470 & 1 & 0.225 & 2.745 \\
\begin{tabular}{c} 
Constant \\
\hline
\end{tabular} & -2.121 & 0.564 & 14.139 & 1 & 0.000 & 0.120 \\
\hline
\end{tabular}

$B$ estimates for the slope coefficients of the univariate logistic regression model containing only this variable, S.E. the estimated standard error for the coefficient of the slope, $D f$ degree of freedom, Wald Wald statistic, $\operatorname{Exp}(B)$ estimates for odds ratio

the level of 0.25 should be the chosen in this operation. These authors have demonstrated that using a traditional level (0.05) often fails to identify variables known to be "scientifically" relevant. As an example of this, we highlight the decision to include the variable genetic test on the construction of the model in our study. Moreover, we emphasize the principles of these authors in modelling - "The analyst, not the comput$\mathrm{er}$, is ultimately responsible for the review and evaluation of the model". In fact, accurate prediction of an individual's future risk for a certain disease could enable the comparison of benefits and risks of treatment, the costs of alternative prevention strategies and selection of the most efficient strategy for the individual. Although studies are subject to methodological difficulties/deficiencies, associations of chronic maxillary sinusitis (CRS) and polymorphisms in more than 30 genes have been published [2]. While the individual risk conferred by these single nucleotide polymorphisms remains modest, taken as a group, they suggest an important implication of pathways

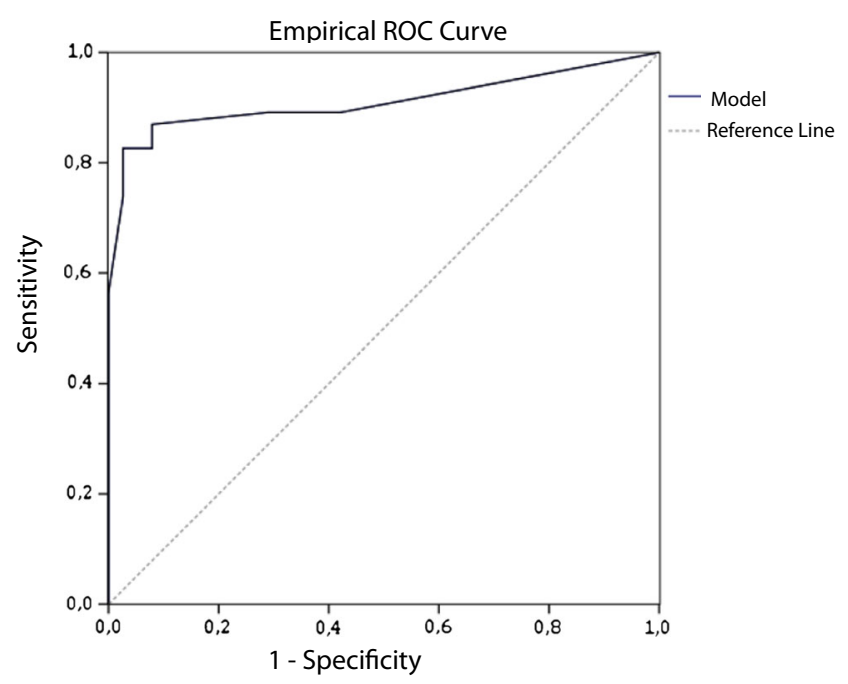

Fig. 4 Empirical ROC curve to the decision model of innate immune recognition and in regulation of downstream signalling in the development of maxillary sinusitis.

Taken individually, no single gene has emerged as uniquely causative of CRS. Although certain studies have reported high risk ratios, enthusiasm for these must be tempered by the small group size involved and the authors' reporting of a genotypespecific effect, rather than the allelic one commonly used. Second, very few of the SNPs identified have been in regions capable of altering amino acid sequences and secreted proteins. Nevertheless, a potentially interesting pattern does emerge. When the identified SNPs are modelled using ingenuity pathway analysis software, a gene-centric network around the TNF, IL1A and NF- $\kappa B$ genes becomes evident, suggesting a central role of this network in the pathology [2]. In 2006, Anand et al. had already reported that there is overexpression of the major genes of the inflammatory pathway (IL-6, IL-12A, IL-13 and $T N F-\alpha)$ in patients with CRS compared with the healthy population. Defining gene expression profiles may help elucidate new key factors in the pathogenesis of CRS and perhaps aid in the development of new therapeutic modalities [33].

This provision may become especially important in implant procedures, usually placed in regions affected by tooth loss, periodontal disease, trauma and oral disease [34, 35].

As an example, the decision to extract a tooth with deficient endodontic treatment, affected with a periapical lesion, or to maintain it, may be evaluated with additional information about the prediction of the inflammatory response of the patient.

In extreme cases, after long-term edentulism, only a thin bone lamella separates the maxillary sinus from the oral cavity. In these cases, the placement of dental implants or procedures of maxillary sinus elevation for rehabilitation may become a challenging situation for the oral surgeon [1].

Several techniques are used to overcome this obstacle, such as bicortical anchorage of dental implants, short implants and sinus floor elevation [9]. If the implant penetrates more than $4 \mathrm{~mm}$, the mucosa of the sinus floor does not spontaneously cover the implant. Then, the implant may act as a foreign body and turns into an inflammatory focus of the maxillary sinus [26].

In a study performed with a population of 206 patients with severe chronic rhinosinusitis, three SNPs in the IRAK4 gene were detected - rs1461567, rs4251513 and rs4251559 — and associated with the increase of the total IgE serum levels $(p<0.004)$. These results demonstrate a clear association between the IRAK4 gene polymorphisms and IgE serum levels in patients with CRS and asthma [36]. Therefore, IRAK4 may play an important role in IgE levels' regulation in patients with inflammatory diseases of the airways. However, in our investigation, we did not find association between the IRAK4 gene studied polymorphism and the presence of asthma. Perhaps 
this occurred because we did not study any of those three polymorphisms referred in the Tewfik study.

Literature studies correlating polymorphisms in the IRAK4 gene with chronic maxillary sinusitis or even with maxillary sinusitis of odontogenic origin are scarce $[2,14]$. However, the genome role importance in this disease has been increasingly evident and some studies even point to a relationship, as is the case of Zhang's (2011) study. The latter reported that the haplotype analysis GCCTGCGA was significantly associated with allergic rhinitis [14].

Another study proposed a genetic predisposition to chronic sinusitis in the Japanese population, concluding that there was an association between $T N F-\beta$ gene polymorphism and chronic sinusitis [37].

The work performed by Sutherland and co-workers was the first report of an association of IRAK4 gene polymorphisms with the prevalence and type of bacterial cultures in a cohort of critically ill patients, supported by mechanistic evidence of decreased cellular immune response to TLR ligands. Their finding that the IRAK4 C/T/A clade was associated with decreased lymphoblastoid cell immune response to $\mathrm{CpG}$ dinucleotide $(\mathrm{CpG}$ - shorthand for " - C - phosphate - G-", cytosine and guanine are separated by only one phosphate) and with a trend to decreased immune response to LPS in IDFs transfected with the IRAK4 expression plasmid carrying the 29429A allele suggests that the replacement of Ala428 with a threonine decreases the cellular response to TLR ligands, perhaps impairing the host's ability to clear an infection [13]. Before that, Picard et al. proposed thatIRAK4 deficiencies predispose patients to recurrent life-threatening bacterial diseases, such as invasive pneumococcal disease, in infancy and early childhood, with weak signs of inflammation. This deficiency was associated with the innate immune response with time; hence, it is no longer so recurrent and fatal [38].

These results, together with the knowledge that the oral flora responsible for odontogenic infections is the same implicated in odontogenic maxillary sinusitis (that is, mixed aerobic and anaerobic flora), lead one to think about the high risk that maxillary sinus is exposed to, after insult with a bacteria-laden displaced tooth root [10]. Add to this, genetic susceptibility to developing infections by Gram-positive bacteria, in the presence of that specific gene polymorphism. So, the whole scene increases the likelihood of developing maxillary sinus pathology, with exacerbated symptoms and characteristics. Perhaps, this IRAK4 gene polymorphism may amplify the response of the innate immunity to Gram-positive bacteria, thus increasing the cytokines production and leading to a more severe inflammatory state of the nasal/sinus mucosa. Hence, our study may be give rise to an initial tool (decision model), to be improved in order to help the diagnosis and the therapeutic decisions in such or similar cases.

Demonstration of a genetic association does not prove causality, which must be established by subsequent experiments showing the altered function of the gene and mechanistic impact in the disease.

Besides this, we conclude that the constructed model could be an initial primitive clinical tool. We are conscientious that chronic diseases are the result of the interaction of several factors. Most of them are even very distant from our assessment. However, if the general dentists and oral surgeons could have improved models, based on the primitive one we presented, probably it could assist in the diagnosis and treatment decision concerning procedures involving maxillary sinus. We emphasize that this primitive model obtained good results by the ROC curve analysis, but this does not mean that they are valid in any population. Thus, we consider that more studies are needed, with more risk factors, with other genes, in other populations, in order to construct more complete decision models, with better representativeness. The present work is only a primitive starting point.

Conflict of interest The authors declare that they have no conflict of interest.

\section{References}

1. Mehra P, Jeong D (2009) Maxillary sinusitis of odontogenic origin. Curr Allerg Asthma Rep 9:238-243

2. Mfuna-Endam L, Zhang Y, Desrosiers MY (2011) Genetics of rhinosinusitis. Curr Allerg Asthma Rep 11:236-246

3. Payne SC, Borish L, Steinke JW (2011) Genetics and phenotyping in chronic sinusitis. J Allerg Clin Immunol 128:710-720

4. Puglisi S et al (2011) Bacteriological findings and antimicrobial resistance in odontogenic and non-odontogenic chronic maxillary sinusitis. J Med Microbiol 60:1353-1359

5. Bomeli SR, Branstetter BFT, Ferguson BJ (2009) Frequency of a dental source for acute maxillary sinusitis. Laryngoscope 119:580 584

6. Kretzschmar DP, Kretzschmar JL (2003) Rhinosinusitis: review from a dental perspective. Oral Surg Oral Med Oral Pathol Oral Radiol Endod 96:128-135

7. Maloney P, Doku H (1968) Maxillary sinusitis of odontogenic origin. J Can Dent Assoc 34:591

8. Paju S, Bernstein JM, Haase EM, Scannapieco FA (2003) Molecular analysis of bacterial flora associated with chronically inflamed maxillary sinuses. J Med Microbiol 52:591-597

9. Galindo-Moreno P, Padial-Molina M, Avila G, Rios HF, HernándezCortés P, Wang HL (2012) Complications associated with implant migration into the maxillary sinus cavity. Clin Oral Implants Res 23: $1152-1160$

10. Mehra P, Murad H (2004) Maxillary sinus disease of odontogenic origin. Otolaryngol Clin North Am 37:347-364

11. Ebenfelt A (2005) Bacterial location in chronic sinusitis. Am J Rhinol 19:458-461

12. Bachert $\mathrm{C}$ et al (1998) The role of cytokines in infectious sinusitis and nasal polyposis. Allergy 53:2-13

13. Sutherland AM, Walley KR, Nakada T-A, Sham AH, Wurfel MM, Russell JA (2011) A nonsynonymous polymorphism of IRAK4 associated with increased prevalence of Gram-positive infection and decreased response to Toll-like receptor ligands. J Innate Immun 3: $447-458$ 
14. Zhang Y et al (2011) Association pattern of interleukin-1 receptorassociated kinase-4 gene polymorphisms with allergic rhinitis in a Han Chinese population. PLoS ONE 6:e21769

15. Chapel H, Puel A, Von Bernuth H, Picard C, Casanova J-L (2005) Shigella sonnei meningitis due to interleukin-1 receptor-associated kinase- 4 deficiency: first association with a primary immune deficiency. Clin Infect Dis 40:1227-1231

16. Medvedev AE et al (2003) Distinct mutations in IRAK-4 confer hyporesponsiveness to lipopolysaccharide and interleukin-1 in a patient with recurrent bacterial infections. J Exp Med 198:521-531

17. Picard $C$ et al (2003) Pyogenic bacterial infections in humans with IRAK-4 deficiency. Science 299:2076-2079

18. Suzuki N, Suzuki S, Yeh W-C (2002) IRAK-4 as the central TIR signaling mediator in innate immunity. Trends Immunol 23:503-506

19. Vaz P, Gallas M, Braga A, Sampaio-Fernandes J, Felino A, Tavares P (2012) IL1 gene polymorphisms and unsuccessful dental implants. Clin Oral Implants Res 23:1404-1413

20. Williams S, Hughes T, Adler C, Brook A, and Townsend G (2014) Epigenetics: a new frontier in dentistry. Aust Dent $\mathrm{J}$

21. Pinheiro A, Facer G, Kern E (1998) Sinusitis: current concepts and management. In: BAILEY, BJ Head and Neck SurgeryOtolaryngology, $2^{\text {nd }}$. Lippincott-Raven Publishers, Philadelphia USA, pp 441-55

22. Abrahams JJ, Glassberg RM (1996) Dental disease: a frequently unrecognized cause of maxillary sinus abnormalities? Am J Roentgenol 166:1219-1223

23. Lopatin AS, Sysolyatin SP, Sysolyatin PG, Melnikov MN (2002) Chronic maxillary sinusitis of dental origin: is external surgical approach mandatory? Laryngoscope 112:1056-1059

24. Carmeli G, Artzi Z, Kozlovsky A, Segev Y, Landsberg R (2011) Antral computerized tomography pre-operative evaluation: relationship between mucosal thickening and maxillary sinus function. Clin Oral Implants Res 22:78-82

25. Connor S, Chavda S, Pahor A (2000) Computed tomography evidence of dental restoration as aetiological factor for maxillary sinusitis. J Laryngol Otol 114:510-513

26. Jung J-H, Choi B-H, Jeong S-M, Li J, Lee S-H, Lee H-J (2007) A retrospective study of the effects on sinus complications of exposing dental implants to the maxillary sinus cavity. Oral Surg Oral Med Oral Pathol Oral Radiol Endodontol 103:623-625

27. Maillet M, Bowles WR, Mcclanahan SL, John MT, Ahmad M (2011) Cone-beam computed tomography evaluation of maxillary sinusitis. J Endod 37:753-757

28. Mélen I, Lindahl L, Andréasson L, Rundcrantz H (1986) Chronic maxillary sinusitis: definition, diagnosis and relation to dental infections and nasal polyposis. Acta Otolaryngol 101:320-327

29. Timmenga NM, Raghoebar GM, Boering G, Van Weissenbruch R (1997) Maxillary sinus function after sinus lifts for the insertion of dental implants. J Oral Maxil Surg 55:936-939

30. Hosmer DW, Lemeshow S (2000) Applied logistic regression, 2nd edn. Wiley, USA, pp 90-142

31. Zou KH, O'Malley AJ, Mauri L (2007) Receiver-operating characteristic analysis for evaluating diagnostic tests and predictive models. Circulation 115:654-657

32. Braga AC, Vaz P, Sampaio-Fernandes JC, Felino A, Tavares MP (2012) Decision model to predict the implant success, in computational science and its applications, ICCSA 2012. Springer, Berlin Heidelberg, pp 665-674

33. Anand VK, Kacker A, Orjuela AF, Huang C, Manarey C, Xiang J (2006) Inflammatory pathway gene expression in chronic rhinosinusitis. Am J Rhinol 20:471-476

34. Garaicoa C, et al. (2013) Using cone beam computed tomography angle for predicting the outcome of horizontal bone augmentation. Clin Implant Dent Relat Res

35. Patel NA, Ferguson BJ (2012) Odontogenic sinusitis: an ancient but under-appreciated cause of maxillary sinusitis. Curr Opin Otolaryngol Head Neck Surg 20:24-28

36. Tewfik MA et al (2009) Polymorphisms in interleukin-1 receptorassociated kinase 4 are associated with total serum IgE. Allergy 64: 746-753

37. Takeuchi K, Majima Y, Sakakura Y (2000) Tumor necrosis factor gene polymorphism in chronic sinusitis. Laryngoscope 110:17111714

38. Picard C et al (2010) Clinical features and outcome of patients with IRAK-4 and MyD88 deficiency. Medicine 89:403-425 